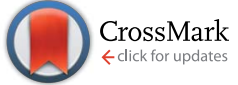

Cite this: Anal. Methods, 2015, 7, 10359
Received 23rd October 2015 Accepted 27th October 2015

DOI: 10.1039/c5ay02790j

www.rsc.org/methods

\section{Large scale preparation of surface enhanced Raman spectroscopy substrates based on silver nanowires for trace chemical detection $\uparrow$}

\begin{abstract}
Qiang Wang, ${ }^{a}$ Xingchen Zhao, ${ }^{b}$ Zhuanni Yu, ${ }^{a}$ Ruiqin $\operatorname{Tan}^{\mathrm{c}}$ and Jing Lan*a
In this paper, a facile large-scale preparation of surface enhanced Raman spectroscopy (SERS) substrates based on silver nanowires has been developed. The SERS substrates can be easily and precisely obtained by filtering bulky amounts of silver nanostructures through hydrophilic filter membranes one time. The developed membranes can be expanded to commercial wafer-style filter membranes of large size (>47 mm in diameter). The as-prepared SERS substrates presented good uniformity and good performance for the detection of crystal violet (CV) and 1,2-di(4-pyridyl)ethylene (BPE). The relative standard deviations (RSD) were less than $5.5 \%(n=11)$ and $5.2 \%(n=11)$ for CV and BPE, respectively. The logarithm of characteristic SERS intensity plotted against CV and BPE concentrations presented a linear relationship over the ranges from $1.0 \times 10^{-4}$ to $1.0 \times 10^{-8} \mathrm{~mol} \mathrm{~L}^{-1}$ and from $5.0 \times 10^{-4}$ to $5.0 \times$ $10^{-9} \mathrm{~mol} \mathrm{~L}^{-1}$, respectively. In the detection of natural water samples of river water, Membrane Bio-Reactor (MBR) effluent and sewage disposal plant effluent, spiked with CV and BPE, the as-prepared SERS substrates also presented good performance, suggesting that such substrates possessed great potential in a broad range of analytical applications.
\end{abstract}

\section{Introduction}

For detecting trace chemicals in various environmental and biological samples, combining chromatography techniques with series of detectors including powerful mass spectrometry has been one of the most routine methods. However, their pretreatment process prior to instrument analysis is often labor intensive, time consuming and costly.

Surface enhanced Raman spectroscopy (SERS) has emerged as an attractive alternative for chemical and biological analysis. $^{\mathbf{1}-6}$

The enhanced signals can be attributed to the surface plasmon resonance (SPR) of the connected nanostructures and the field enhancement. ${ }^{7-10}$ And it has been reported that Raman signals of the analytes can be optimized by selecting shape/sizecontrollable metallic nanoparticles. ${ }^{\mathbf{1 0}}$

In a typical procedure, a SERS analysis involves spotting a $\mu \mathrm{L}$-volume of sample onto a nanofabricated SERS substrate, drying and then detecting the Raman scattering. Unfortunately,

\footnotetext{
${ }^{a}$ College of Chemistry and Pharmaceutical Sciences, Qingdao Agricultural University, Qingdao 266109, China. E-mail: qdlanjing@mail.sdu.edu.cn; Tel: +86-532-8608-0894 ${ }^{b}$ State Key Laboratory of Environmental Chemistry and Ecotoxicology, Research Center for Eco-Environmental Sciences, Chinese Academy of Sciences, P.O. Box 2871, Beijing 100085, China

${ }^{c}$ Ningbo University, Ningbo, China

$\dagger$ Electronic supplementary information (ESI) available. See DOI: 10.1039/c5ay02790j
}

the widespread utilization of SERS has often been prevented by the high cost and inconvenience, in part, as a result of the complicated fabrication of SERS-active substrates. Therefore, a lot of efforts on fabricating SERS substrates have continued to be made, such as improving the interactions between substrates and compounds, and fabricating shape-controllable metallic nanostructures., ${ }^{\mathbf{9 1 1 - 1 4}}$ The most widely used substrates are metallic nanoparticles which can be prepared in a wide size distribution range and in various shapes. Recently, some commercial carriers have been introduced to simplify the fabrication process of SERS-active substrates and reduce the cost of these developed substrates. ${ }^{15-18}$ For example, Yu et al. have developed inkjet-printed SERS substrates on paper ${ }^{16}$ and SERS membranes based on silver nanoparticles filtration. ${ }^{\mathbf{1 9}}$ Very recently, Shi et al. have combined extraction and SERS scattering together by using a hydrophilic filter membrane loaded with silver nanowires. ${ }^{20}$ These efforts have provided a new and convenient strategy to prepare SERS substrates. Nevertheless, large scale fabrications of SERS substrates are still time consuming and their reproducibility is suspicious as a result of the smaller size of the carriers or multiple preparation processes.

In this paper, size-controllable silver nanowires have been fabricated for preparing the SERS substrates by filtering them through large-size filter membranes. During the typical process, bulky amounts of silver nanowires can be homogenously trapped on the large-size filter membrane by a single filtration step, and the thickness of the silver layer can be controlled by adjusting the amount of silver nanowires. To evaluate the 
potential of the prepared SERS substrates in routine analysis, the uniformity, the reproducibility and the performance on natural water samples have been studied by analyzing CV and BPE.

\section{Experimental}

\section{Chemicals}

Silver nitrate $\left(\mathrm{AgNO}_{3}, 99.8 \%\right)$ and ethanol were purchased from Sinopharm Chemical Reagent Co. (Beijing, China). Ethylene glycol (EG) was purchased from BDH. Polyvinylpyrrolidone $(\mathrm{PVP}, \mathrm{MW}=55000 \mathrm{~g})$ was purchased from Sigma. Mixed cellulose membranes ( $47 \mathrm{~mm}$ diameter and $0.22 \mu \mathrm{m}$ pore size) were purchased from Millipore. Ultra-pure water $(18.25 \mathrm{M} \Omega \mathrm{cm})$ was used throughout the experiments.

\section{Synthesis of silver nanowires}

The preparation of silver nanowires was modified from the method developed by Mahmoud et al. ${ }^{21}$ In a typical procedure, $70 \mathrm{~mL}$ of EG was added into a glass flask and refluxed at $140{ }^{\circ} \mathrm{C}$ under stirring for $1 \mathrm{~h}$. Then the temperature of the EG solution was increased to $175^{\circ} \mathrm{C}$ and $0.5 \mathrm{~g}$ PVP was added. The stirring rate was increased to $1600 \mathrm{rpm}$ and a solution containing $0.15 \mathrm{~g}$ $\mathrm{AgNO}_{3}$ and $3 \mathrm{~mL}$ EG was injected. The mixed solution was kept stirring until the color of the solution turned from brownish, opaque yellow to olive. To terminate the growth of silver nanowires, the mixture was immediately transferred into a beaker cooled with water-ice solution and the stirring speed continued to be maintained at $1600 \mathrm{rpm}$. The length of the silver nanowires can be controlled by adjusting the reaction time. The prepared silver nanowires were separated and purified 3 times with ultra-pure water and ethanol separately. An appropriate precipitate dried under $\mathrm{N}_{2}$ protection was dispersed into ultra-pure water as stock solutions for further SERS activated substrate preparation.

\section{Fabrication of the silver nanowire membranes}

The SERS-active substrates based on silver nanowires were prepared by a flow-through method. In brief, the hydrophilic filter membrane was fixed on a massive volume glass filter (Fig. 1). Different volumes of stock colloid solution of silver nanowires were diluted to $500 \mathrm{~mL}$ and then added into the holder of the glass filter. The diameter of the quartz sand support vector of the glass filter was $40.0 \mathrm{~mm}$. Then the silver nanowire colloid was passed through the filter membrane under the assistance of an air extraction pump. Finally, the silver nanowires were trapped on the filter membrane and emerged as the SERS-active substrates. The thickness of the trapped silver nanowire membranes can be controlled by adjusting the amount of silver nanowires. The prepared SERSactive substrates were cut into segments with an area of $3.0 \times$ $3.0 \mathrm{~mm}^{2}$ for subsequent SERS analysis.

\section{Substrate characterization}

The morphology of the as-prepared SERS substrates was obtained with a field-emission electron microscope (SU8020,

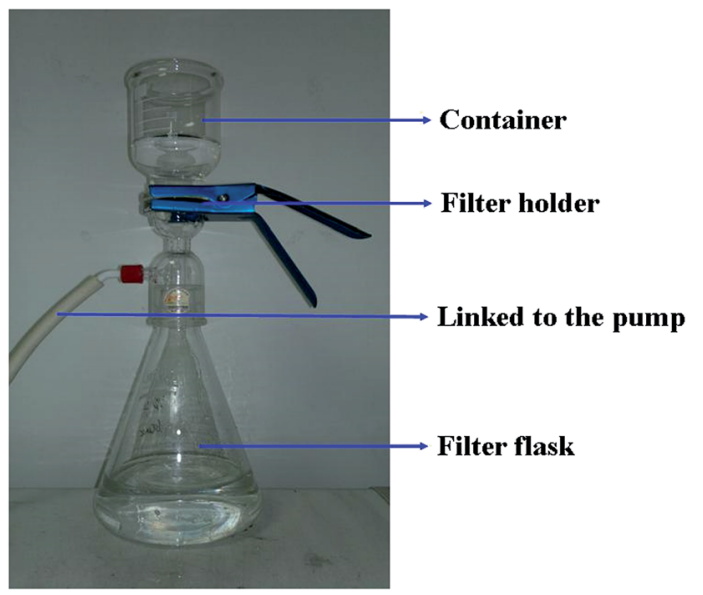

Fig. 1 Large-scale SERS-active membranes are created by passing certain volumes of silver nanowire colloid solution through a hydrophilic filter membrane wafer under the assistance of a pump. To obtain good uniformity, the velocity of the solution can be controlled by using peristaltic pump.

Hitachi, Japan) with an accelerating voltage of $3 \mathrm{kV}$. The X-ray diffraction (XRD) patterns were recorded with a PANalytical X'Pert Pro X-ray diffractometer (PANalytical, Netherland) equipped with Al K-Alpha radiation $(40 \mathrm{kV}, 200 \mathrm{~mA})$.

\section{SERS assay}

Appropriate volumes of sample solutions were dropped on the surface of SERS-active substrates with silver nanowires and dried in air naturally. Then the SERS spectra were recorded by using a Renishaw InVia Raman microscope equipped with 532, 633 and $785 \mathrm{~nm}$ laser lines as the excitation sources. The laser power was maintained at $1 \mathrm{~mW}$ throughout the experiment and the acquisition time was $5 \mathrm{~s}$. The reproducibility was evaluated by Raman mapping of a randomly selected more than $14 \times$ $19 \mu \mathrm{m}^{2}$ area.

All the obtained SERS spectra were background subtracted and the signal intensities used for calculation were extracted from these subtracted SERS spectra.

\section{Results and discussion}

\section{Characterization of silver nanowires}

Fig. 2a shows that this SERS-active membrane consisted of interwoven uniform silver nanowires ( $\sim 40 \mathrm{~nm}$ in diameter), which will enable a large enhancement when compared to conventional Raman spectroscopy. Fig. $2 \mathrm{~b}$ and c present the digital image and magnified SEM image of the prepared SERSactive membrane. Compared with uniform silver nanowires (Fig. 2d), disordered nanowires were trapped on the membrane. The thickness of silver nanowires membrane can also be controlled by adjusting the amount of silver nanowires. The powder XRD pattern shows that these silver nanowires maintained a cubic-phase Ag structure, corresponding to the standard JCPDS card no. 040783 (Fig. 2e), which indicated that the silver nanowires were finely crystallized. 

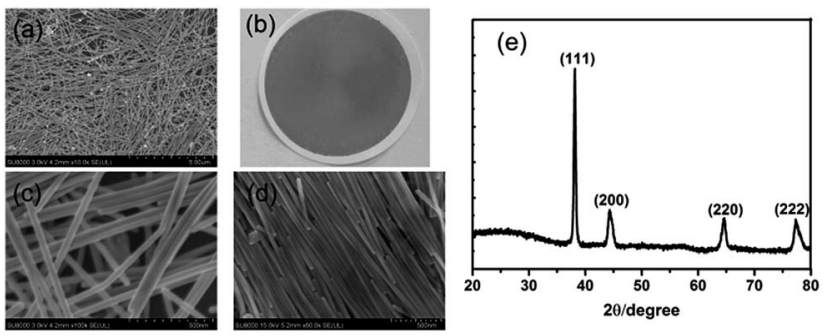

Fig. 2 (a) SEM image of silver nanowires trapped on the filter membrane; (b) digital image of the prepared SERS-active membrane; (c) magnified SEM image of silver nanowires trapped on the filter membrane; (d) SEM image of fresh silver nanowires; (e) XRD patterns of silver nanowires.

\section{Effect of the amount of loaded silver nanowires on their SERS performance}

Intuitively, the amount of silver nanowires loaded on the surface of the filter membrane will affect the SERS activity, as only a certain amount of silver nanowires is able to create a high surface density but loading too many will lead to a relatively thick silver film instead of a plasmonic nanostructured surface. Since CV and BPE have distinct Raman features and they can be easily adsorbed onto the nanostructured surface, both of them have been used as probe molecules to evaluate the prepared SERS-active membrane in this study. The optimal laser wavelength is $633 \mathrm{~nm}$ for these both molecules (ESI $\dagger$ ). Fig. 3a and b show SERS spectra of CV and BPE adsorbed on the developed SERS-active membrane with different amounts of silver nanowires. The signal intensity increased significantly until the amount of silver nanowires was $c a .0 .6 \mathrm{mg} \mathrm{cm}^{-2}$ and then began to remain constant. The results suggested that the performance of the SERS-active membrane could be optimized and repeated, as the thickness of the nanostructured membrane could be easily and precisely controlled by adjusting the amount of silver nanowires. The developed SERS-active membrane loaded with more silver nanowires presented higher reproducibility with RSDs of less than $5.5 \%(n=11)$ and $5.2 \%(n=11)$ for CV and $\mathrm{BPE}$, respectively. When the number of probe molecules is insufficient to form a monolayer on the silver nanostructured surface, the thickness increase of the silver membrane will give rise to higher surface area and lower surface coverage, and then
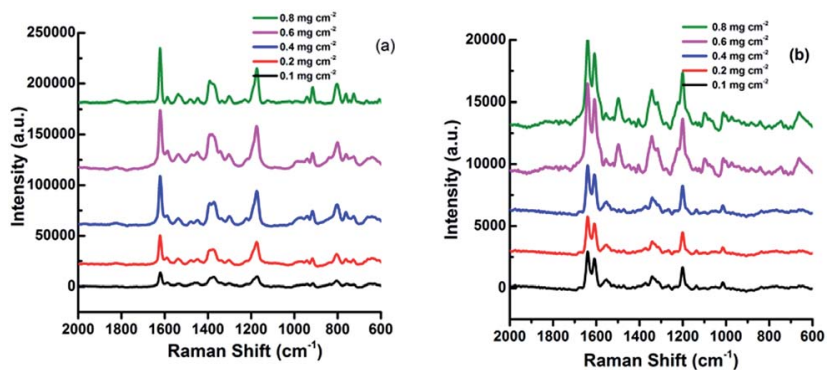

Fig. 3 (a) SERS spectra of CV on the developed membrane. (b) SERS spectra of BPE on the developed membrane. (The concentrations of probe molecules are $1.0 \times 10^{-6} \mathrm{~mol} \mathrm{~L}^{-1}(\mathrm{CV})$ and $5.0 \times 10^{-6} \mathrm{~mol} \mathrm{~L}^{-1}$ (BPE)). lead to the reduction of molecules being illuminated by the signal intensity of laser. Therefore, the SERS-active membrane with $0.6 \mathrm{mg} \mathrm{cm}^{-2}$ silver layer was chosen for further evaluation of its performance.

\section{Surface enhancement Raman scattering characterization}

The SERS-active membrane based on silver nanowires could be easily cut or tailored into any desired shape for routine SERS analysis. In this paper, the prepared membranes were cut into slices with an area of $0.3 \times 0.3 \mathrm{~cm}^{2}$ for further SERS analysis. To test the limit of detection (LOD) of the developed SERS-active membrane based on silver nanowires, as well as to quantify the Raman enhancement effect, the Raman spectra of CV and BPE with different concentrations were recorded. Fig. 4a and b show the Raman spectra of $\mathrm{CV}$ and BPE with the concentrations from $1.0 \times 10^{-4}$ to $1.0 \times 10^{-8} \mathrm{~mol} \mathrm{~L}^{-1}$ and from $5.0 \times 10^{-4}$ to $5.0 \times$ $10^{-9} \mathrm{~mol} \mathrm{~L}^{-1}$, respectively. Data points representing the height of the $1640 \mathrm{~cm}^{-1} \mathrm{CV}$ Raman peak and the height of the $1608 \mathrm{~cm}^{-1}$ BPE Raman peak were plotted against CV and BPE concentrations in Fig. $4 \mathrm{c}$ and $\mathrm{d}$, respectively. In the plots, the data points represented the mean value of three separate membranes, and the value associated with each membrane was the mean value of three different spots on the membrane. For comparison, the spectra from normal Raman spectroscopy (NRS) of CV $\left(1.0 \times 10^{-2} \mathrm{~mol} \mathrm{~L}^{-1}\right)$ and BPE $\left(5.0 \times 10^{-2} \mathrm{~mol} \mathrm{~L}^{-1}\right)$ were also collected, and these results are also presented in Fig. $4 \mathrm{a}$ and $\mathrm{b}$. It is evident that the Raman spectrum was discriminable when the concentrations of CV and BPE dropped to $1.0 \times 10^{-8}$ and $5.0 \times 10^{-9} \mathrm{~mol} \mathrm{~L}^{-1}$, respectively.

The Raman enhancement factor (EF) is estimated by comparing the measured SERS intensity of $\mathrm{CV}\left(1640 \mathrm{~cm}^{-1}\right)$ or
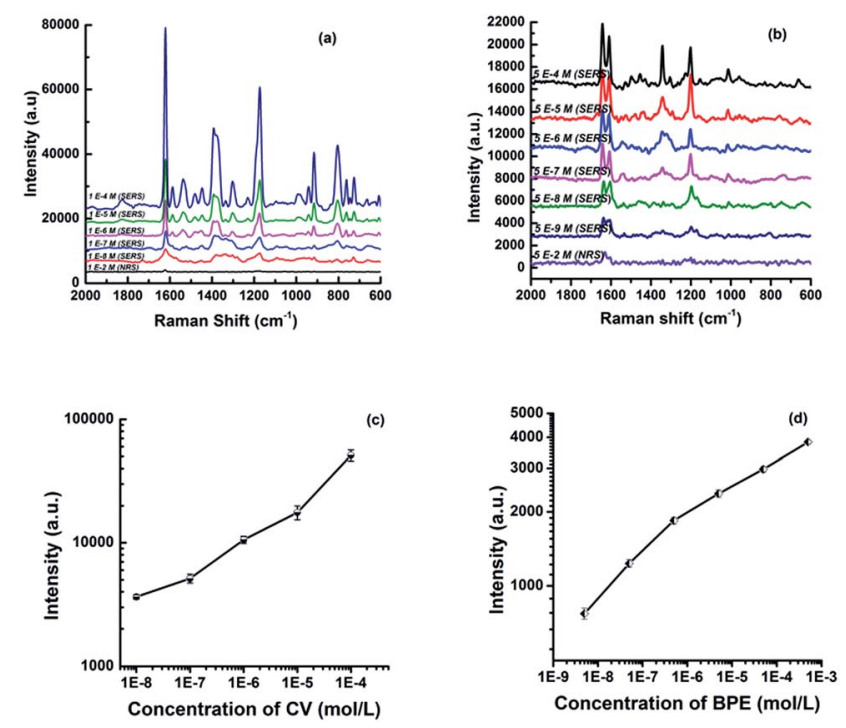

Fig. 4 (a) SERS spectra of different concentrations of CV on the developed SERS-active membrane. (b) SERS spectra of different concentrations of BPE on the developed SERS-active membrane. (c) Raman intensity at $1640 \mathrm{~cm}^{-1}$ as a function of the CV concentration. (d) Raman intensity at $1608 \mathrm{~cm}^{-1}$ as a function of the BPE concentration. 
BPE $\left(1608 \mathrm{~cm}^{-1}\right)$ on the developed SERS-active membrane and its normal Raman intensity by using the following equation:

$$
\mathrm{EF}=\left(\frac{I_{\text {SERS }}}{I_{\text {Raman }}}\right)\left(\frac{N_{\text {Raman }}}{N_{\text {SERS }}}\right)
$$

where $I$ is the height of the $1640 \mathrm{~cm}^{-1} \mathrm{CV}$ Raman peak or the height of the $1608 \mathrm{~cm}^{-1}$ BPE Raman peak; $N$ is the number of $\mathrm{CV}$ or BPE molecules under laser radiation (see the details in the $\mathrm{ESI} \dagger$ ). As the SERS intensity did not increase linearly with the probe molecule concentration (shown in Fig. 4c and d), the calculated EF using different concentrations should be different. In this paper, the calculated EFs for CV and BPE were in the range of $1.8 \times 10^{4}$ to $7.7 \times 10^{6}$ and of $1.0 \times 10^{5}$ to $1.4 \times$ $10^{6}$, respectively. The results were comparable with the reported value $\left(10^{5}\right.$ to $\left.10^{6}\right)$ in the literature..$^{19,20,22-26}$ The enhancement may be attributed to abundant Raman hotspots such as nanopores/nanogaps and the junctions between NWs. ${ }^{\mathbf{8 , 2 7 , 2 8}}$

\section{Uniformity and stability of the developed SERS-active membrane}

The uniformity, stability and reproducibility of the active substrate are crucial for SERS detection, ${ }^{29-31}$ as Raman scattering can provide fingerprint information by analyzing vibrational and rotational modes of a system. For evaluating the uniformity of the developed SERS-active membrane, point-bypoint Raman mappings were recorded on a randomly selected $14 \times 19 \mu \mathrm{m}^{2}$ area for CV and BPE with a step size of $1 \mu \mathrm{m}$. The SERS Raman intensity mappings at $1640 \mathrm{~cm}^{-1}$ for $\mathrm{CV}$ and $1608 \mathrm{~cm}^{-1}$ for BPE are shown in Fig. 5a and b, presenting a homogeneous distribution of the Raman intensities of these characteristic peaks. Fig. $5 \mathrm{c}$ and d show the Raman spectra collected from the strongest points (point A and $\mathrm{C}$ ) and the weakest points (point B and D). It was found that the difference on SERS intensities was also at a comparatively low level between these strongest and weakest points. The distributions of occurrence of Raman intensities for CV $\left(1640 \mathrm{~cm}^{-1}\right)$ and BPE $\left(1608 \mathrm{~cm}^{-1}\right)$ presented that more than $90 \%$ of the total points were concentrated in the vicinity of median intensity (Fig. 5e and $\mathrm{f}$ ). The RSDs of $\mathrm{CV}$ and BPE were $7.2 \%$ and $6.8 \%$ for all those detected points (266 points), respectively. Therefore, the results from Raman mapping further confirmed the good uniformity of the developed SERS-active membrane.

To evaluate the temporal stability of the SERS-active membrane, the SERS spectra of CV and BPE were also recorded under continuous laser irradiation for 200 seconds. No obvious change on the overall shape was observed, though there were a few small changes on the SERS intensity with RSDs of $5.8 \%$ and $5.2 \%$ for CV and BPE, respectively. This indicated that the SERS-active membrane possessed good temporal stability under continuous laser irradiation, enabling its usage for routine SERS analysis.

\section{Performance of the developed SERS-active membrane in natural water samples}

To evaluate the potential of the developed SERS-active membrane for routine analysis, a certain amount of probe (a)
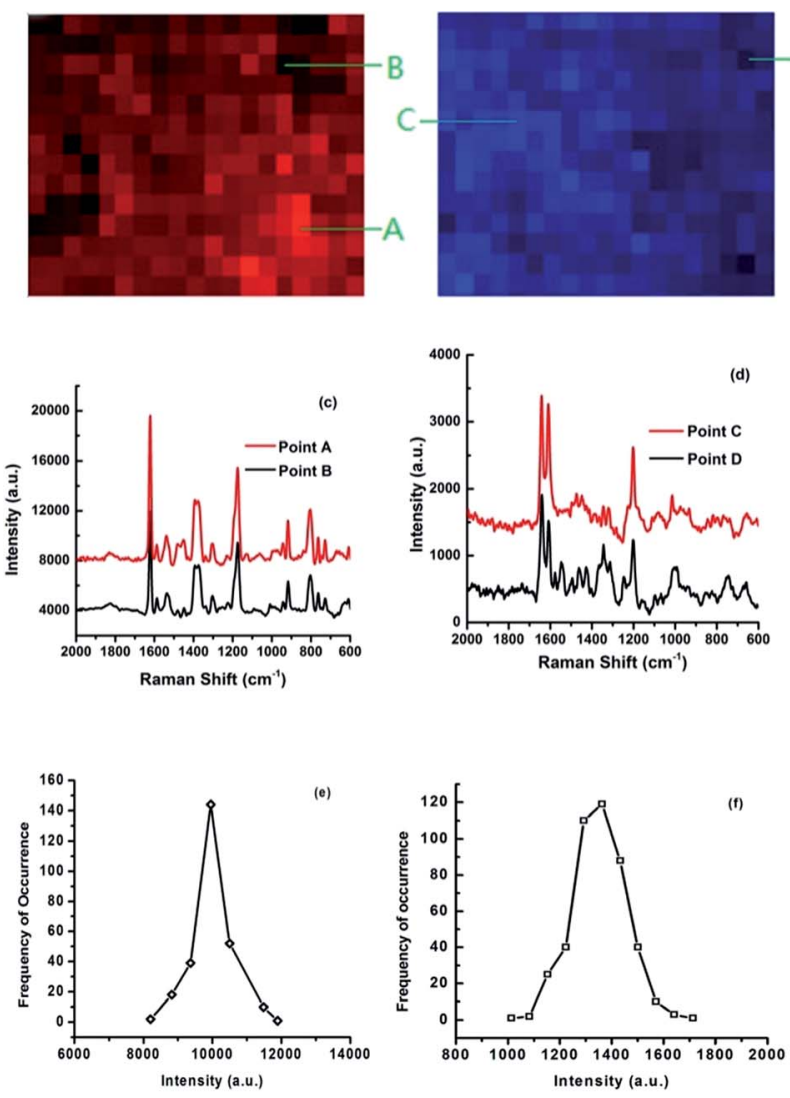

Fig. 5 (a and b) SERS intensity maps of CV at $1640 \mathrm{~cm}^{-1}$ and BPE at $1608 \mathrm{~cm}^{-1}$, respectively (each grid represents $1.0 \times 1.0 \mu \mathrm{m}^{2}$ ). (c and d) The Raman intensity of the strongest and weakest points for $\mathrm{CV}$ and $B P E$, respectively. (e and f) Distributions of frequency of occurrence of Raman intensities at 1640 and $1608 \mathrm{~cm}^{-1}$ for $C V$ and BPE, respectively.

molecules was added into several natural water samples and further used to evaluate the performance of these developed membranes. $\mathrm{CV}$, a hazardous purple dye in textiles and paints, and BPE, a widely used chemical intermediate, were chosen as the probe molecules due to their good SERS signals. The concentrations of $\mathrm{CV}$ and BPE were $1.0 \times 10^{-6}$ and $5.0 \times 10^{-6}$ mol L ${ }^{-1}$, respectively. The natural water samples included two river water samples (R1 and R2), one effluent water sample from a membrane bioreactor (MBR) (M1) and one effluent water sample from a wastewater treatment plant (T1). The Raman spectra of these natural water samples with certain concentrations of $\mathrm{CV}$ and BPE added are recorded in Fig. $6 \mathrm{a}$ and $\mathrm{b}$. The Raman spectra of these initial water samples without these probe molecules were also recorded as blank for comparison. It is obvious that the Raman signals of water samples in the presence of probe molecules could be markedly enhanced. Although there were quite a few interfering signals, their peaks close to characteristic peaks of probe molecules were very small and their effects on the determination could be neglected. When comparing with the SERS spectra of the standard solutions with the same concentrations (Fig. 4a and b), the intensities were comparable to each other. When simply calculated by using the standard curves, the concentrations in T1, M1, R1 

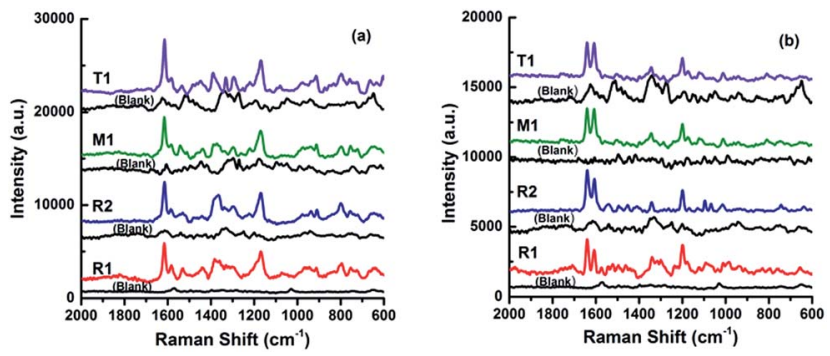

Fig. 6 (a) SERS spectra of natural water samples with and without the addition of CV. (b) SERS spectra of natural water samples with and without the addition of BPE. Blank represents no probe molecule addition. The concentrations of $\mathrm{CV}$ and BPE spiked into the water samples are $1.0 \times 10^{-6}$ and $5.0 \times 10^{-6} \mathrm{~mol} \mathrm{~L}^{-1}$, respectively.

and R2 are $0.86-1.1 \times 10^{-6} \mathrm{~mol} \mathrm{~L}^{-1}$ for $\mathrm{CV}$ (recoveries of $86-110 \%$ ) and $4.5-5.6 \times 10^{-6} \mathrm{~mol} \mathrm{~L}^{-1}$ for BPE (recoveries of 90-112\%), respectively. Additionally, the RSDs of CV and BPE in these natural water samples were $6.1-7.8 \%$ and $7.2-9.4 \%(n=$ 6 ), respectively. This implied that these developed SERS-active membranes could be used for routine SERS analysis in environmental investigation.

\section{Conclusions}

In summary, a facile technique to fabricate a SERS-active membrane based on silver nanowires in larger scale has been demonstrated. In the typical fabrication protocol, lots of silver nanowires were trapped onto the hydrophilic filter membrane of large size by filtering the colloids of these silver nanostructures. The thickness of the developed membrane could be controlled by adjusting the amount of silver nanowires. The SERS results suggested that the amount of nanowires trapped on the surface of the filter membrane was critical for enhancing SERS signals. The developed SERS-active membrane presented good uniformity and temporal stability. The technique for preparing SERS substrates with high sensitivity, reproducibility and stability on a large scale may be a good choice for routine SERS analysis.

\section{Acknowledgements}

The authors thank Professor Liu Jingfu and Doctor Liu Rui for the technical assistance. This work was supported by the National Natural Science Foundation (Grant numbers 21407086 and 21477138) and Doctor Fund of Qingdao Agricultural University (63311330 and 63311203).

\section{References}

1 J. Kneipp, H. Kneipp and K. Kneipp, Chem. Soc. Rev., 2008, 37, 1052-1060.

2 Y. Li, L. Qu, D. Li, Q. Song, F. Fathi and Y. Long, Biosens. Bioelectron., 2013, 43, 94-100.

3 L. Qian and R. Mookherjee, Nano Res., 2011, 4, 1117-1128.
4 C. Zhu, G. Meng, Q. Huang and Z. Huang, J. Hazard. Mater., 2012, 211-212, 389-395.

5 B. Liu, G. Han, Z. Zhang, R. Liu, C. Jiang, S. Wang and M. Han, Anal. Chem., 2012, 84, 255-261.

6 V. Joseph, C. Engelbrekt, J. Zhang, U. Gernert, J. Ulstrup and J. Kneipp, Angew. Chem., Int. Ed., 2012, 51, 7592-7596.

7 X. Jiang, Y. Lai, M. Yang, H. Yang, W. Jiang and J. Zhan, Analyst, 2012, 137, 3995-4000.

8 S. Lee, A. Morrill and M. Moskovits, J. Am. Chem. Soc., 2006, 128, 2200-2201.

9 L. Jensen, C. M. Aikens and G. C. Schatz, Chem. Soc. Rev., 2008, 37, 1061-1073.

10 H. J. Chen, X. S. Kou, Z. Yang, W. H. Ni and J. F. Wang, Langmuir, 2008, 24, 5233-5237.

11 D. He, B. Hu, Q. F. Yao, K. Wang and S. H. Yu, ACS Nano, 2009, 3, 3993-4002.

12 Z. M. Zhang, J. F. Liu, R. Liu, J. F. Sun and G. H. Wei, Anal. Chem., 2014, 86, 7286-7292.

13 J. Hu, M. Tanabe, J. Sato, K. Uosaki and K. Ikeda, J. Am. Chem. Soc., 2014, 136, 10299-10307.

14 R. Liu, J. F. Liu, X. X. Zhou, M. T. Sun and G. B. Jiang, Anal. Chem., 2011, 83, 9131-9137.

15 C. H. Lee, M. E. Hankus, L. M. Tian, P. M. Pellegrino and S. Singamaneni, Anal. Chem., 2011, 83, 8953-8958.

16 W. W. Yu and I. M. White, Anal. Chem., 2010, 82, 9626-9630. 17 Y. J. Meng, Y. C. Lai, X. H. Jiang, Q. Q. Zhao and J. H. Zhan, Analyst, 2013, 138, 2090-2095.

18 Q. S. Mei and Z. P. Zhang, Angew. Chem., Int. Ed., 2012, 51, 5602-5606.

19 W. W. Yu and I. M. White, Analyst, 2012, 137, 1168-1173.

20 Y. E. Shi, L. Li, M. Yang, X. Jiang, Q. Zhao and J. Zhan, Analyst, 2014, 139, 2525-2530.

21 M. A. Mahmoud, M. A. El-Sayed, J. P. Gao and U. Landman, Nano Lett., 2013, 13, 4739-4745.

22 H. J. Chen, Y. L. Wang, J. Y. Qu and S. J. Dong, J. Raman Spectrosc., 2007, 38, 1444-1448.

23 Z. N. Zhu, H. F. Meng, W. J. Liu, X. F. Liu, J. X. Gong, X. H. Qiu, L. Jiang, D. Wang and Z. Y. Tang, Angew. Chem., Int. Ed., 2011, 50, 1593-1596.

24 D. Cialla, U. Hubner, H. Schneidewind, R. Moller and J. Popp, ChemPhysChem, 2008, 9, 758-762.

25 R. Alvarez-Puebla, B. Cui, J. P. Bravo-Vasquez, T. Veres and H. Fenniri, J. Phys. Chem. C, 2007, 111, 6720-6723.

26 W. B. Cai, B. Ren, X. Q. Li, C. X. She, F. M. Liu, X. W. Cai and Z. Q. Tian, Surf. Sci., 1998, 406, 9-22.

27 H. Ko, S. Singamaneni and V. V. Tsukruk, Small, 2008, 4, 1576-1599.

28 R. Gunawidjaja, S. Peleshanko, H. Ko and V. V. Tsukruk, Adv. Mater., 2008, 20, 1544-1549.

29 R. H. Que, M. W. Shao, S. J. Zhuo, C. Y. Wen, S. D. Wang and S. T. Lee, Adv. Funct. Mater., 2011, 21, 3337-3343.

30 L. L. Qu, D. W. Li, J. Q. Xue, W. L. Zhai, J. S. Fossey and Y. T. Long, Lab Chip, 2012, 12, 876-881.

31 L. L. Qu, Y. T. Li, D. W. Li, J. Q. Xue, J. S. Fossey and Y. T. Long, Analyst, 2013, 138, 1523-1528. 